\title{
International Diabetes Federation document concerning postmeal glycemic control: assessment of quality of clinical practice guidelines using AGREE instrument
}

\author{
Jan Brożek ${ }^{1-3}$, Miłosz Jankowski ${ }^{1}$, Ewa Płaczkiewicz-Jankowska ${ }^{3}$, Roman Jaeschke ${ }^{3,4}$ \\ 1 Department of Internal Medicine, Jagiellonian University, Medical College, Kraków, Poland \\ 2 Department of Epidemiology, Italian National Cancer Institute "Regina Elena", Rome, Italy \\ 3 Polish Institute for Evidence Based Medicine, Kraków, Poland \\ 4 Department of Medicine, McMaster University, Hamilton, ON, Canada
}

\section{KEY WORDS}

AGREE instrument, assessment of guidelines, clinical practice guidelines, diabetes, postprandial glycemia
Correspondence to: Mitosz Jankowski, MD, PhD, II Katedra Chorób Wewnętrznych, Uniwersytet Jagielloński, Collegium Medicum ul. Skawińska 8, 31-066 Kraków, phone: + 48-12-29-34-294 fax: + 48-12-293-40-30, e-mail:mjankow@mp.pl Received: August 4, 2008. Revision accepted: October 7, 2008 Conflict of interest: none declared. Pol Arch Med Wewn. 2009; 119 (1-2): 18-24

Translated by Professional Language Services SIGILLUM Ltd., Kraków Copyright by Medycyna Praktyczna, Kraków 2009

\begin{abstract}
Clinical practice guidelines constitute one of the most important sources of information and education for physicians. Therefore, establishing rules to develop and appraise such guidelines properly is of increasing importance. This task is served by the AGREE (Appraisal of Guidelines Research and Evaluation) instrument, a questionnaire, which, according to its authors, allows reproducible assessment of guideline quality. The aim of this paper is to allow readers familiarize themselves with such rules of guidelines appraisal. In order to achieve this purpose, we present the actual application of the AGREE instrument using as an example recently published document on postmeal hyperglycemia issued by the International Diabetes Federation.
\end{abstract}

INTRODUCTION Clinical practice guidelines (CPG) are becoming one of the basic educational and informational tools for physicians. Scientific associations and other organizations, whether local, national or international, develop such documents, expecting their members and other users of the guidelines to act according to their recommendations. Compliance with CPG recommendations is sometimes used as an index of health care quality, and the number of CPG is increasing. It is not infrequent that CPG addressing the same clinical situations are issued independently by different organizations. For example, physicians interested in treatment of venous thromboembolism may take advantage of current documents issued by American College of Chest Physicians ${ }^{1}$, American College of Physicians/American Academy of Family Physicians ${ }^{2}$, American Society of Clinical Oncology ${ }^{3}$, Institute for Clinical Systems Improvement ${ }^{4}$, International Union of Angiology in cooperation with other international scientific associations ${ }^{5}$, National Comprehensive Cancer
Network ${ }^{6}$ and National Institute for Health and Clinical Excellence ${ }^{7}$. Recommendations included in individual CPG are frequently similar, but sometimes differ. There are numerous reasons for which various scientific associations or organizations develop independent documents dealing with similar clinical situations, starting from local conditions, like availability of drugs, common prevention methods, etc, through the needs of specific groups and organizations, to differences of opinions.

It is important for users, i.e. physicians, nurses, physiotherapists, patients and other interested parties, to have an opportunity to recognize and select CPG with the best odds of benefiting patients to whom CPG are applied. However, such choice is difficult, because it is not easy to assess which guidelines are "the best". Many publications do not even allow the reader to be precisely aware to whom they are directed to, what evidence serve as the basis for recommendations or what was the association between the authors 
and the manufacturers of recommended drugs or devices. The need to establish standard criteria for guideline assessment has been recognized by an international group of physicians, researchers and those employed in various health care sectors interested in methodology CPG development. The AGREE (Appraisal of Guidelines Research and Evaluation) instrument is the result of their cooperation. ${ }^{8}$ This instrument is a questionnaire, which, according to its authors, allows reproducible quality assessment of CPG.

The main objective of this paper is to review the rules of assessment and development of guidelines designed to influence clinical practice. It is neither a systematic nor comprehensive review, but more an attempt to introduce the AGREE instrument to readers and demonstration of its practical use for assessment of the recently published document concerning postmeal hyperglycemia issued by the International Diabetes Federation (IDF) and available on the IDF website, recently also in Polish (www.idf.org). Its Polish version has also been published in the monthly Medycyna Praktyczna. ${ }^{9}$ The International Diabetes Federation encompasses over two hundred other associations and organizations involved in diabetes therapy, rates among the most reputable and respected diabetic organizations, and its objective is education, prevention and treatment of diabetes in the world.

AGREE instrument The objective of the AGREE instrument is to establish a system for critical appraisal of guidelines. ${ }^{8}$ In this framework clinical practice guidelines are systematically developed statements with aim to assist practitioners and patients in deciding appropriate management in specific clinical circumstances. ${ }^{10}$ Their purpose is to make explicit recommendations with a definite intent to influence what clinicians do. ${ }^{11}$

Quality of CPG is meant by the authors of the AGREE instrument as a degree of confidence that: 1) appropriate steps have been taken during the process of guideline development to avoid an error leading to formulation of untrue recommendations, 2) the recommendations are applicable to patients, whose data have been used to establish the recommendations, and to similar (but potentially slightly different) patients, to whom the recommendations may apply in the future, and 3) there is a possibility of introduction of a given recommendation to clinical practice. According to the AGREE, when assessing the CPG quality, benefits, harms and costs resulting from following individual recommendations and associated practical issues should be considered. Therefore, a critical look involves assessment of methods, final wording of individual recommendations and consideration of factors, which may influence introduction of the guidelines into practice.

The AGREE instrument may be used for assessment of CPG dealing with any clinical situation. It may be used by health service managers, CPG developers, physicians and health care providers attempting to independently assess the quality of guidelines before making decisions about implementing their recommendations, and by those involved in education within a given field of medicine.

The AGREE consists of 23 key items organized in 6 domains. Each domain pertains to a separate dimension of CPG quality. The first one, scope and purpose ( 3 items), is to assess the precision of the clinical questions. The second one pertains to stakeholder involvement (4 items) and assesses the extent to which the guideline represents the views of its intended users. The following group of seven items (rigor of CPG development) relates to the process used to gather and synthesize the evidence, the method to formulate the recommendations and to update them. The fourth domain (clarity and presentation 4 items) deals with the language and format. The following domain (applicability, 3 items) assesses the organizational, behavioural and cost implications of guideline application. The last covered domain (2 items) is independence, defining the extent of independence of recommendations and acknowledgement of possible conflict of interest from the guideline development group.

Answers to the questions concerning individual quality criteria are rated on a 4-point scale (from 1 - "strongly disagree", through 2 - "disagree", 3 "agree" to 4 - "strongly agree"). Although the assessment results in individual domains could be found helpful while comparing quality of different guidelines, it is impossible to determine the threshold score for each domain which recognizes CPG as "beneficial" or "not beneficial".

To illustrate process described above we present the use of AGREE instrument for the appraisal of document dealing with postprandial glycemic control issued by IDF. Individual items of the AGREE instrument are discussed one by one with clarification of their significance and our assessment of the extent to which the IDF guidelines meet a given criterion.

Scope and purpose

1 General purpose of CPG has been precisely defined.

1.1 AGREE clarification: this deals with the potential health impact of a guideline on society and populations of patients. The overall objective(s) of the guideline should be described in detail and the expected health benefits from the guideline should be specific to the clinical problem.

1.2 IDF guidelines: according to the developers of the analyzed guidelines, the purpose of this guideline is to present data from reports that describe the relationship between postmeal glucose and the development of diabetic complications. Based on these data, recommendations for the appropriate management of postmeal glucose in type 1 and type 2 diabetes have been developed. The recommendations are intended to assist 
clinicians and organizations in developing strategies to effectively manage postmeal glucose in patients with type 1 and type 2 diabetes, taking into consideration locally available therapies and resources. Postmeal glycemic control in pregnancy has not been addressed in this guideline.

1.3 Our assessment: 4 points (strongly agree).

2 The clinical question(s) covered by the guideline is(are) specifically described

2.1 AGREE clarification: a detailed description of the clinical questions covered by the guideline should be provided, particularly for the key recommendations (see item 17).

2.2 IDF guidelines: "As a basis for developing the recommendations, the Guideline Development Group addressed four questions relevant to the role and importance of postmeal hyperglycemia in diabetes management. The evidence supporting the recommendations is shown as evidence statements (with the level of evidence indicated at the end of the statement)." Those questions were as follows: 1) Is postmeal hyperglycemia harmful? 2) Is treatment of postmeal hyperglycemia beneficial? 3) Which therapies are effective in controlling postmeal plasma glucose? 4) What are targets for postmeal glycemic control and how should they be assessed?

2.3 Our assessment: 4 points (strongly agree). 3 The patients to whom the guideline is meant to apply are specifically described.

3.1 AGREE clarification: there should be a clear description of the target population to be covered by a guideline. The age range, sex, clinical description, comorbidity may be provided.

3.2 IDF guidelines, according to their developers, should deal with postmeal glycemia both in type 1 and type 2 diabetic patients, but do not apply in pregnancy.

3.3 Our assessment: 3 (agree).

\section{Stakeholder involvement}

4 The guideline development group includes individuals from all the relevant professional groups.

4.1 AGREE clarification: this item refers to the professionals who were involved at some stage of the development process. This may include members of the steering group, the research team involved in selecting and reviewing/rating the evidence and individuals involved in formulating the final recommendations. This item excludes individuals who have externally reviewed the guideline (see item 13). Information about the composition, discipline and relevant expertise of the guideline development group should be provided.

4.2 IDF guidelines: "The process involved a broadly based group of people, including patients with diabetes, healthcare professionals from diverse disciplines and people from nongovernmental organizations. The project was overseen by the Steering Committee (...) and input was provided by the entire Guideline Development Group (individual persons specified in the document). The Guideline Development Group included people with considerable experience in guideline development, healthcare development and delivery, and people living with diabetes. Geographical representation included all IDF regions and countries in different states of economic development.

4.3 Our assessment: 3 (agree, no details).

5 The patients' views and preferences have been sought.

5.1 AGREE clarification: information about patients' experiences and expectations of health care should inform the development of clinical guidelines.

5.2 IDF guidelines: the developers mentioned as follows: "The process involved a broadly based group of people, including people with diabetes (...)."

5.3 Our assessment: 3 (agree, no details)

6 The target users of the guideline are clearly defined.

6.1 AGREE clarification: the target users should be clearly defined in the guideline, so they can immediately determine if the guideline is relevant to them.

6.2 IDF guidelines: "The recommendations are intended to assist clinicians and organizations in developing strategies to effectively manage postmeal glucose in people with type 1 and type 2 diabetes, taking into consideration locally available therapies and resources. (...) Logic and clinical judgment remain critical components of diabetes care and implementation of the guideline recommendations."

6.3 Our assessment: 3 (agree, no details).

7 The guideline has been piloted among target users.

7.1 AGREE clarification: a guideline should have been pre-tested for further validation amongst its intended end users prior to publication. This process should be documented.

7.2 IDF guidelines: no data available.

7.3 Our assessment: 2 (disagree, no data available).

Rigor of development

8 Systematic methods were sued to search for evidence.

8.1 AGREE clarification: "Details of the strategy used to search for evidence should be provided including search terms used, sources consulted and dates of the literature covered. Sources may include electronic databases (e.g. MEDLINE, EMBASE, CINAHL), databases of systematic reviews (e.g. the Cochrane Library, DARE), journals, conference proceedings and other guidelines (e.g. the US National Guideline Clearinghouse, the German Guidelines Clearinghouse)."

8.2 IDF guidelines: "The evidence used in developing this guideline included reports from key meta-analyses, evidence-based reviews, clinical trials, cohort studies, epidemiological studies, animal and basic science studies, position statements and guidelines (English language only). 
A scientific writer with knowledge of diabetes obtained relevant reports through a computerized search of available data using the PubMed and other search engines; scanning incoming journals in the medical library and reviewing the references in pertinent s, major textbooks and syllabi from national and international meetings on the subjects of diabetes, using relevant title and text words (e.g. postprandial, postmeal, hyperglycemia, mealtime, self-monitoring, oxidative stress, inflammation) as search criteria. Evidence relating to both postmeal and postchallenge plasma glucose was reviewed and cited as appropriate. A review of recent guidelines, position statements and recent articles not identified in the universal search was also conducted to obtain additional information that was potentially applicable to the questions. An electronic database was created to include full reference information for each report; abstracts for most of the reports were included in the database. Members of the Steering Committee were asked to identify any additional reports or publications relevant to the questions. In total, 1,659 reports were identified."

8.3 Our assessment: 4 (strongly agree).

9 The criteria for selecting the evidence are clearly described.

9.1 AGREE clarification: criteria for including/ excluding evidence identified by the search should be provided. These criteria should be explicitly described and reasons for including and excluding evidence should be clearly stated. For example, guideline authors may decide to only include evidence from randomised clinical trials and to exclude articles not written in English.

9.2 IDF guidelines: the authors used all available evidence and based their recommendations on those with highest quality. For this purpose they used the evidence-grading criteria system developed by the Scottish Intercollegiate Guidelines Network (SIGN, see TABLE; for more information on methods see the document on: www. idf.org).

Note: the evidence-grading criteria system mentioned above gives the highest ranks to randomized controlled trials, which pertain mainly to therapeutical questions and lower to prognosis trials, which were the basis for the answer to question number 1.

9.3 Our assessment: 4 (strongly agree).

10 The methods used for formulating the recommendations are clearly described.

10.1 AGREE clarification: there should be a description of the methods used to formulate the recommendations and how final decisions were arrived at. Methods include, among others, a voting system, formal consensus techniques (e.g. Delphi, Glaser techniques). Areas of disagreement and methods of resolving them should be specified.

10.2 IDF guidelines: "The Guideline Development Committee met to discuss the evidence statements and supporting data and to develop the recommendations. A recommendation was made according to the level of scientific substantiation based on evidence ratings whenever possible. However, when there was a lack of supporting studies, the Steering Committee formulated a consensus recommendation."

10.3 Our assessment: 3 (agree)

11 The health benefits, side effects and risks have been considered in formulating the recommendations.

11.1 AGREE clarification: the guideline should consider health benefits, side effects, and risks of the recommendations. These may include: survival, quality of life, adverse effects, and symptom management or a discussion comparing one treatment option to another. There should be evidence that these issues have been addressed.

11.2 IDF guidelines: each recommendation was accompanied by an extensive description of evidence and a set of evidence-based statements which constituted the the basis for the recommendations.

11.3 Our assessment: 3 (agree, however, no discussion on costs and non-monetary expenses incurred by a patient).

12 There is an explicit link between the recommendations and the supporting evidence.

12.1 AGREE clarification: there should be an explicit link between the recommendations and the evidence on which they are based. Each recommendation should be linked with a list of references on which it is based.

12.2 IDF guidelines: a link between the recommendations and the evidence, on which they were based, was clearly identified in the text.

12.3 Our assessment: 3 (agree formally, recommendation for treatment not resulting directly from the presented evidence).

13 The guideline has been externally reviewed by experts prior to its publication.

13.1 AGREE clarification: a guideline should be reviewed externally before it is published. Reviewers should not have been involved in the development group and should include some experts in the clinical area and some methodological experts. Patients' representatives may also be included. A description of the methodology used to conduct the external review should be presented, which may include a list of the reviewers and their affiliation.

13.2 IDF guidelines: "The evidence cited to support the recommendations was reviewed by two independent external reviewers who were not part of the Guideline Development Committee. Comments from the external reviewers were then reviewed by the Steering Committee." "The draft guideline was sent out for a wider external review to IDF member associations, global and regional IDF elected representatives, interested professionals, industry and others on IDF contact lists, for a total of 322 invitations. Thirty-eight comments from 20 external reviewers from five of the seven IDF regions (Africa, South East Asia, Western Pacific, North America, Europe) were received. These 


\begin{tabular}{l}
\hline $1+$ High-quality meta-analyses, systematic reviews of RCTs, or RCTs with a very low risk of bias \\
\hline $1+$ Well-conducted meta-analyses, systematic reviews of RCTs, or RCTs with a low risk of bias \\
\hline $1-$ Meta-analyses, systematic reviews of RCTs, or RCTs with a high risk of bias \\
\hline $2++$ High-quality systematic reviews of case-control or cohort studies; \\
\hline high-quality case control or cohort studies with a very low risk of confounding bias and a high probability that the relationship is causal \\
\hline $2+\quad$ Well-conducted case-control or cohort studies with a low risk of confounding bias or chance and a moderate probability that \\
the relationship is causal; well-conducted basic science with a low risk of bias \\
\hline $2-\quad$ Case-control or cohort studies with a high risk of confounding bias or chance and a significant risk that the relationship is not causal \\
\hline 3 Non-analytic studies (for example s, case series) \\
\hline 4 Expert opinion
\end{tabular}

Abbreviations: RCT - randomized controlled trials

comments were reviewed by the Steering Committee and considered in developing the final document."

13.3 Our assessment: 4 (agree).

14 A procedure for updating the guideline is provided.

14.1 AGREE clarification: guidelines need to reflect current research. There should be a clear statement about the procedure for updating the guideline. For example, a timescale has been given, or a standing panel receives regularly updated literature searches and makes changes as required.

14.2 IDF guidelines: IDF considers review and update within three years.

14.3 Our assessment: 3 (agree, although the update intent is not precise).

\section{Clarity and presentation}

15 The recommendations are specific and unambiguous.

15.1 AGREE clarification: a recommendation should provide a concrete and precise description of which management is appropriate in which situation and in what patient group, as permitted by the body of evidence. However, evidence is not always clear cut and there may be uncertainty about the best management. In this case the uncertainty should be stated in the guideline.

15.2 IDF guidelines: below we present recommendations corresponding to the four main questions:

Question 1 Is postmeal hyperglycemia harmful? Recommendation 1 Postmeal hyperglycemia is harmful and should be addressed.

Question 2 Is treatment of postmeal hyperglycemia beneficial?

Recommendation 2 Implement treatment strategies to lower postmeal plasma glucose in patients with postmeal hyperglycemia.

Question 3 Which therapies are effective in controlling postmeal plasma glucose?

Recommendation 3 A variety of both non-pharmacologic and pharmacologic therapies should be considered to target postmeal plasma glucose.

Question 4 What are targets for postmeal glycemic control and how should they be assessed?
Recommendations 4 Two-hour postmeal plasma glucose should not exceed $7.8 \mathrm{mmol} / 1$ (140 mg/dl) as long as hypoglycemia is avoided.

Self-monitoring of blood glucose should be considered because it is currently the most practical method for monitoring postmeal glycemia.

Efficacy of treatment regimens should be monitored as frequently as needed to guide therapy towards achieving the postmeal plasma glucose target.

15.3 Our assessment: 2 (disagree; see Additional comments).

16 The different options for management of the condition are clearly presented.

16.1 AGREE clarification: a guideline should consider the different possible options for screening, prevention, diagnosis or treatment of the condition it covers.

16.2 IDF guidelines: as stated above, a number of treatment options described by the authors cover almost every hyperglycemia control method.

16.3 Our assessment: 4 (agree).

17 Key recommendations are easily identifiable.

17.1 AGREE clarification: users should be able to find the most relevant recommendations easily. These recommendations answer the main clinical questions that have been covered by the guideline. They can be identified in different ways. For example, they can be summarised in a box, typed in bold, underlined or presented as flow charts or algorithms.

17.2 IDF guidelines: the above mentioned suggestions have been fulfilled.

17.3 Our assessment: 4 (agree).

18 The guideline is supported with tools for application.

18.1 For a guideline to be effective it needs to be disseminated and implemented with additional materials. These may include for example, a summary document, a quick reference guide, educational tools, leaflets for patients, computer support.

18.2 IDF guidelines: no materials mentioned above.

18.3 Our assessment: 2 (disagree; see Additional comments). 
Applicability

19 The potential organizational barriers in applying the recommendations have been discussed.

19.1 AGREE clarification: applying the recommendations may require changes in the current organisation of care within a service or a clinic which may be a barrier to using them in daily practice. Organisational changes that may be needed in order to apply the recommendations should be discussed.

19.2 IDF guidelines: taking into account the low level of precision and explicitness of recommendations, the above aspects have not been considered.

19.3 Our assessment: 2 (disagree, not applicable). 20 The potential cost implication of applying recommendations have been considered.

20.1 AGREE clarification: the recommendations may require additional resources in order to be applied. For example, there may be a need for more specialised staff, new equipment, expensive drug treatment. These may have cost implications for health care budgets. There should be a discussion of the potential impact on resources in the guideline.

20.2 IDF guidelines: the cost issue has been treated superficially: "Although cost will remain an important factor in determining appropriate treatments, controlling glycemia is ultimately much less expensive than treating the complications of diabetes."

20.3 Our assessment: 2 (disagree).

21 The guideline presents key review criteria for monitoring and/or audit purposes.

21.1 AGREE clarification: measuring the adherence to a guideline can enhance its use. This requires clearly defined review criteria that are derived from the key recommendations in the guideline.

21.2 IDF guidelines: treatment goals are clearly specified: $\mathrm{HbA}_{1 c}<6.5 \%$, premeal (fasting) plasma glucose $<5.5 \mathrm{mg} / \mathrm{dl}$, 2-hour postmeal plasma glucose $<7.8 \mathrm{mg} / \mathrm{dl}$.

21.3 Our assessment: 3 (agree, although practical aspects of reaching those goals remain uninvestigated).

Editorial independence

22 The guideline is ly independent from the funding body.

22.1 AGREE clarification: some guidelines are developed with external funding (e.g. Government funding, charity organisations, pharmaceutical companies). Support may be in the form of financial contribution for the whole development, or for parts of it, e.g. printing of the guidelines. There should be an explicit statement that the views or interests of the funding body have not influenced the final recommendations. Please note: If it is stated that a guideline was developed without external funding, then you should answer "strongly agree".

22.2 IDF guidelines: "This activity was supported by unrestricted educational grants from:
Amylin Pharmaceuticals, Eli Lilly and Company, LifeScan, Inc., Merck \& Co. Inc, Novo Nordisk A/S, Roche Diagnostics GmbH, Roche Pharmaceuticals. These companies did not take part in the development of the guideline. However, these and other commercial organizations on IDF's communications list were invited to provide comments on draft versions of the guideline."

22.3 Our assessment: 3 (agree, although the level of authors' independence from the industry is usually much more complex than "no external funding" recommended by the AGREE).

23 Conflict of interest of guideline development members have been recorded.

23.1 AGREE clarification: there are circumstances when members of the development group may have conflicts of interest. For example, this would apply to a member of the development group whose research on the topic covered by the guideline is also funded by a pharmaceutical company. There should be an explicit statement that all group members have declared whether they have any conflict of interest.

23.2 IDF guidelines: members of the Guideline Development Committee have declared relevant dualities of interest in the topic and in relationships with commercial enterprises, governments and non-governmental organizations. No fees were paid to the Guideline Development Committee members in connection with the current activity.

23.3 Our assessment: 3 (agree to a degree similar to other guidelines and documents, probably in the future the authors would be required much more detailed declaration).

Final comments Analysis of IDF guidelines using the AGREE instrument allowed us to make certain observations concerning both documents.

First, the examination of the utility of the AGREE instrument suggests that it should not be used to specifically determine clinical usefulness of CPG or to make unambiguous decisions about adhering to their recommendations. Instead, AGREE should rather be used to identify the domains, which should be more closely considered by both the CPG users and the persons responsible for their update. Numerical scores attributed to individual domains will lack significance without reference to clinical context, which requires thorough expertise. Clinical context is an essential component of the process of CPG development and assessment. Clinical questions themselves, which should be answered by individual recommendations, are of fundamental significance. In the case of the IDF guidelines considered here, clinical questions concern postmeal blood glucose; it could, however, be noted that although the evidence of harm of postmeal hyperglycemia is not in doubt, it has not been demonstrated by authors of IDF guidelines that treatment aiming at its reduction was associated with a decrease in mortality caused by diabetic complications. This observation follows the fact that it 
is quite difficult to separate therapeutical effect associated with treatment of fasting and postmeal hyperglycemia. It should be remembered that maintenance of optimum glycemic control, i.e. both fasting and postmeal, assessed also indirectly using $\mathrm{HbA}_{1 c}$ value, remains the main goal of diabetes treatment. The IDF guidelines are a document of a world-wide application, i.e. addressed to a number of various health care systems, emphasizing the significance of postmeal hyperglycemia treatment that should be considered in management of all diabetic patients.

The third and, in our opinion, the most important observation concerns the understanding of the CPG's role. We believe that it should be a set of recommendations, which assists physicians and other health care providers in making optimal decisions in patient care. The objective of their development is therefore a direct impact on actions of physicians, health care providers and patients. On the other hand the IDF document is mostly a position statement, i.e. a set of statements, saying that (postmeal) hyperglycemia is harmful, that (postmeal) hyperglycemia should be treated using usual methods, and that self-measurement of blood glucose by patients is useful. Irrespective of a general nature of such statements (and not recommendations), the least specific part pertains to treatment of (postmeal) hyperglycemia, where the authors of the IDF document say as follows: "A variety of both non-pharmacologic and pharmacologic therapies should be considered to target postmeal plasma glucose." From the user's (physician, nurse or patient) point of view, precise information concerning selection of particular medications would be more desirable. Lack of precise recommendations for treatment of (postmeal) hyperglycemia is a result of inadequate evidence, which would allow the authors of "guidelines" to determine relative benefits of following various accessible treatment methods and to suggest practical instructions.

In summary one should consider the following question: "Would you recommend application of those guidelines in practice?" The AGREE instrument allows the following options: "strongly recommend", "recommend (with reservations or after amendments)", "not recommend" and "not certain". In our opinion, the IDF "guidelines" concerning postmeal glucose control may be used to emphasize the role of hyperglycemia control, including postmeal hyperglycemia, in diabetes treatment. Unfortunately, they are not helpful when selecting the optimal treatment method, probably since the associated clinical trial evidence is still limited. It seems that physicians and patients, who would like to receive detailed instructions concerning management of postmeal hyperglycemia, have to wait for the following update of the document.

\section{REFERENCES}

1 Kearon C, Kahn SRR, Agnelli G, et al. Antithrombotic therapy for venous thromboembolic disease: American College of Chest Physicians ev idence-based clinical practice guidelines (8th edition). Chest. 2008; 133: S454-S545.

2 Snow V, Qaseem A, Barry P, et al. Management of Venous Thromboembolism: A Clinical Practice Guideline from the American College of Physicians and the American Academy of Family Physicians. Ann Intern Med. 2007; 146: 204-210.

3 Lyman GH, Khorana AA, Falanga A, et al. American Society of Clini cal Oncology Guideline: Recommendations for Venous Thromboembolism Prophylaxis and Treatment in Patients With Cancer. J Clin Oncol. 2007; 25: 5490-5505.

4 Burnett B, Helt J, Larsen J, et al. Institute for Clinical Systems Improve ment. Health Care Guideline: VTE Thromboembolism. Eighth Edition/June 2007. www.icsi.org.

5 Nicolaides AN, Fareed J, Kakkar AK, et al. Prevention and treatment of venous thromboembolism. International Consensus Statement (Guidelines according to scientific evidence). International Angiology. 2006; 25 : 101-161.

6 Wagman LD, Fanikos J, Fogarty PF, et al. NCCN Clinical Practice Guidelines in Oncology. Venous Thromboembolic Disease. Version 1.2007. www. nccn.org.

7 National Collaborating Centre for Acute Care. Venous Thromboembolism. Reducing the risk of venous thromboembolism (deep vein thrombosis and pulmonary embolism) in inpatients undergoing surgery.

8 AGREE Collaboration. Appraisal of Guidelines for Research \& Evaluation (AGREE) Instrument. www.agreecollaboration.org.

9 [Management of postmeal hyperglycemia - Guidelines of the International Diabetes Federation. Based on Guideline for management of postmeal glucose International Diabetes Federation]. Med Prakt. 2008; 203: 74-79. Polish.

10 Lohr KN, Field MJ. A provisional instrument for assessing clinical practice guidelines. In: Field MJ, Lohr KN, eds. Guidelines for clinical practice. From development to use. National Academy Press, Washington D.C., 1992.

11 Hayward RS, Wilson MC, Tunis SR, et al. Users' guides to the Medical Literature. VIII. How to Use Clinical Practice Guidelines. A. Are the Recommendations Valid? JAMA. 1995; 274: 570-574. 